\title{
Vision-Based Grasp Planning and Experiments of a Mobile Manipulator
}

\author{
Yi-Fu Chiu \\ Institute of Electrical Control Engineering, National Chiao Tung University \\ 1001 Ta Hsueh Road, Hsinchu 30010, Taiwan \\ Kai-Tai Song \\ Institute of Electrical Control Engineering, National Chiao Tung University \\ 1001 Ta Hsueh Road, Hsinchu 30010, Taiwan \\ E-mail: ivan790721.ece01g@nctu.edu.tw,ktsong@mail.nctu.edu.tw
}

\begin{abstract}
In this paper, a motion planner is designed and implemented for of a mobile manipulator to travel to a spot for grasping of an object. In this work, the probability of successful grasping inside the workspace of the robot arm is used for grasping planning. A vision SLAM system is combined with reachability calculation to figure out the grasping position. Using a laboratory dual-arm robot, we conducted experiments in different conditions to verify the effectiveness of the developed system.
\end{abstract}

Keywords: Mobile manipulation, visual servoing, visual navigation, robot grasping.

\section{Introduction}

Mobile manipulation technologies have been developed rapidly in recent years. It has been expected that various robots will come into our everyday life for living aids. In order to help people with housework, a robot needs to be equipped with many abilities such as communication, navigation, grasping and object recognition. Therefore, developing a mobile manipulator which is able to move and handle objects such that it can support people in a home setting deserves urgent attention.

Cosero $^{1}$ has achieved notable success in RoboCup@Home contests for its mobile manipulation performance. It localizes objects and plans path based on a 2D occupancy grid map constructed from multiple $3 \mathrm{D}$ scans of the environment ${ }^{2}$. Collision with obstacles can be avoid using the map, even though it cannot perceive an obstacle with current sensor's view. PR2 ${ }^{3}$ processes sensory data from $3 \mathrm{D}$ visual sensors in point clouds. Multilayered 2D costmaps and a layered representation of the robot body are used to reduce the possibility of collision ${ }^{4}$. In the work of Stulp et al. ${ }^{5}$, through experience-based learning, the robot first learns a so-called generalized success model which distinguishes among positions from which manipulation may succeed or fail. The model is used to compute a probability distribution that maps positions to a predicted probability of successful manipulation, taking into account the uncertainty of the robot and object's positions.

A key factor of mobile manipulation design is that a suitable pose of the mobile robot needs to be obtained through navigation in order to conduct object manipulation. One possibility is simply to travel from the current position to a position such that the target object is reachable, as depicted in Fig.1. As shown in Fig 1(a), the object is placed in front of the table, where an obstacle exists to influence the grasping. In this case, the object might not be successfully grasped due to the limited workspace of the robot arm. However, if the mobile robot travels to the side of the table as shown in Fig. 1(b), the object can be easily grasped. It is therefore 
desirable to develop a method to plan a pose for the robot such that the target object is easy-to-reach.

Attamimi et al. ${ }^{6}$ proposed a method to use perception maps that express the robot arm reachability and robot platform navigation reachability to a target object. The authors also show that by integrating these two maps, a proper position that has maximum probability of successful grasping is determined. In this study, we developed a motion planning system for determining the proper pose for grasping objects. The robot position is determined based on the arm's reach and ease of movement. We adopted the design of Attamimi et al. ${ }^{6}$ to describe the limitations of robot workspace, the situation of environment and target object position. By constructing the reachability map that represents the ease of robot mobile platform motion and the graspability map that describes the success rate of grasping of robot arms in workspace, robot is able to determine the proper position to move toward and increase the success rate of mobile manipulation task. Visual EFK-SLAM and visual servo technics are integrated into the design for vision-based grasping of the object.

\section{The Dual Arm Robot}

A dual-arm robot is designed and constructed for mobile manipulation experiments. The 6-DOF dual-arm design allows the robot to grasp various objects in the environment. A Kinect RGBD camera is installed on top of the robot for visual servo control. The forward and inverse kinematics of two robot arms are derived and implemented on the robot controller. Two laser range finders are equipped on the mobile platform in forward and backward directions for obstacles detection. An on-board industrial PC (IPC) is used for data processing and motion control of the robot. The

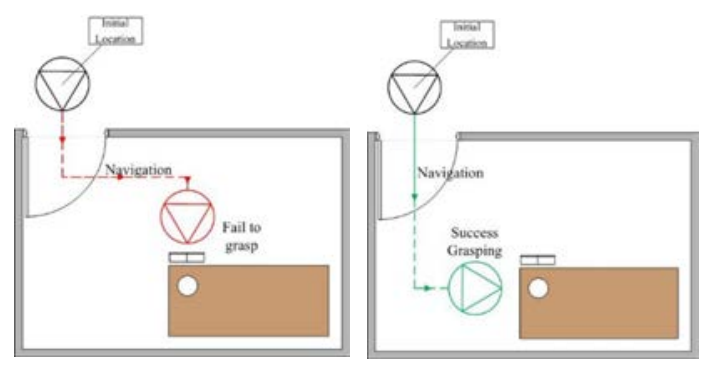

(a)

Fig.1 An example of mobile manipulation is influenced by an obstacle in front of the target. steer- and-drive mechanism allows the robot to move in omnidirection $^{7}$. The implemented EKF-SLAM ${ }^{8}$ allows the robot to navigate and localize itself in the environment. The visual servo system ${ }^{9}$ allows the robot to move and fetch an object without collisions.

\section{A. Visual EKF-SLAM}

A visual SLAM system has been developed for the omnidirectional mobile robot $^{8}$. There are three main steps of the EKF-SLAM: a measurement step to acquire the location vector of robot relative to the feature in the environment, a prediction step to predict the location of robot using motion model and an update step to calculate Kalman gain and update robot stare according to above two steps. Then, the updated robot state is delivered to prediction step to calculate the next moment state of robot. These steps repeat to achieve the real time localization and mapping.

\section{B. Visual servo grasping}

By using the depth data from Kinect, the input image is segmented into several planes. In this $\operatorname{method}^{9}$, the plane that the target object locates is first labeled. The labeled plane is featured by SURF algorithm and is matched with data base to find where the target object is. Except for the target object, the other objects in the environment are obstacle for robot manipulation. Potential field is then combined gravity and repulsion with these indices for safe path planning of robot arms. According to this, the robot is able to move its arms in the workspace without collisions and to grasp the targeted object.

\section{Grasp Planning}

The proposed system architecture is shown in Fig. 2. For representation of the environment, we utilize EKFSLAM algorithm and Kinect sensor to construct a feature map of the environment. For grasping planning, we adopted the method of Attamimi et al. ${ }^{6}$ in this design. The feature map is used as input to the mobile platform to calculate the easiness of traveling to the reachable region of the robot. On the other hand, the target location is inputted to arm graspability calculation to represent the extent of graspable region of robot arms. We fuse the two representations to decide the goal location for robot to grasp the target object. This work uses EKF-SLAM algorithm for robot navigation 

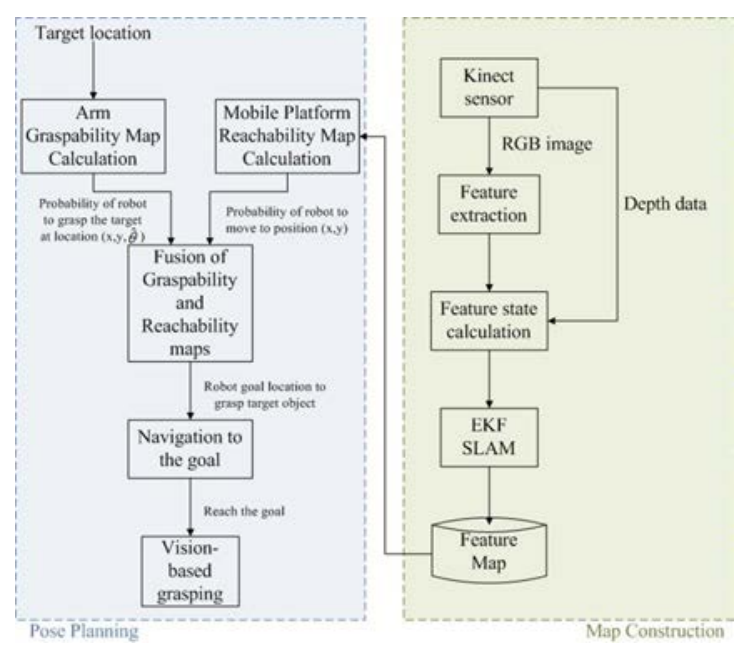

Fig.2 System architecture

control. First, we extract SURF features that represent the configuration of the environment. Then, EKF is utilized to improve the localization error and to correct the coordinates of features. For grasping, we use Kinect for environment detection so that the robot is able to detect not only the target object but also the obstacle in the environment and to guide its arms to fetch the target. The inverse kinematics solution map suggested in the work of Attamimi et al. ${ }^{6}$ is adopted to calculate reachable/unreachable points. Then a probability distribution is given to all reachable points for later decision making of the grasping position.

To include the description of robot navigation in the motion planning of grasping, we have to consider the navigation control of the mobile robot in an environment. Obstacles in the environment influence the motion planning of the robot. In this work, a feature map is built to express the environment by using EKFSLAM algorithm. We adopted the method proposed by Attamimi et al. ${ }^{6}$ to construct a reachability map for motion planning. The closer to obstacles, the less probability is given for the robot to reach. The motion planning of the robot is then determined for the robot to move to the maximum probability of the integrated representation of the navigation and arm motion ${ }^{6}$.

\section{Experiments and discussion}

Three interesting experiments have been carried out for grasp planning to verify the effectiveness of the proposed system. We first verify that different locations of target object in the same environment will result in different robot navigation behavior. Then we add an obstacle around the target area to verify the adaptation of the robot.

\section{A. Target object in different locations}

Fig. 3 shows the environment of this experiment, Condition A and Condition B are termed to distinguish two locations of the object on the table. We first utilize EKF-SLAM to construct the feature map of the environment. Then we replace the features with the size of robot. Fig. 4(a) shows the planning result of condition A. It is noted that the planned grasping position is in front of the table, which is depicted in dotted line. For condition B, the planned grasp position is at the side of the table, as shown in Fig. 4(b). Fig. 5 shows the experiment by real robot in Condition A and Fig. 6 shows the experiment in Condition B.

\section{B. Grasping planning affected by obstacles}

The purpose of this experiment is to verify the proposed method is able to judge the proper grasp position under the obstacle situation. Fig. 7 (a) shows the setup of this experiment, termed Condition C. The target object is at the same position as Condition A but is behind the obstacle. The planning result is shown in Fig. 7(b). The graspable region moves to the side of the table because of the graspability affected by the obstacle changes. Compare with Condition A, the difference is the obstacle effect that causes the different planning from proposed method.

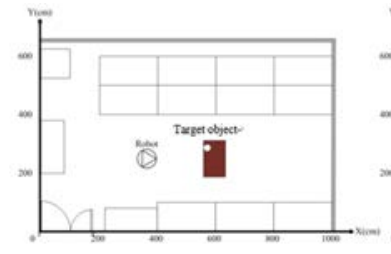

(a)

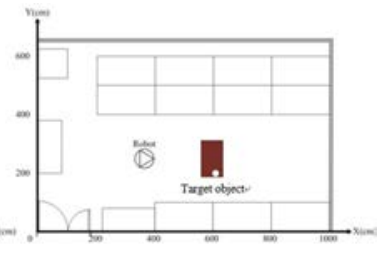

(b)
Fig. 3 Environment setup for experiments (a) Condition A, target in the front of the front of the table, (b) Condition B, target on the side of the table. 


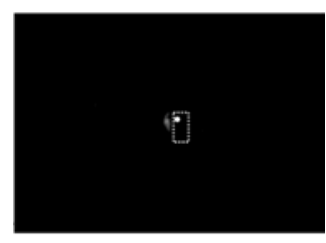

(a)

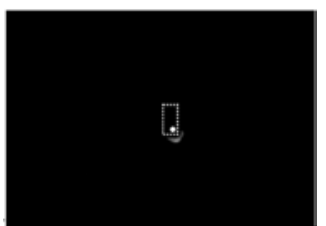

(b)
Fig. 4 (a) The planning result of Condition A, (b) the planning result of Condition B.

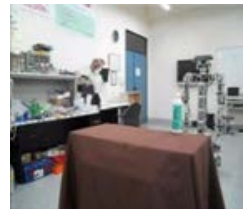

(a)

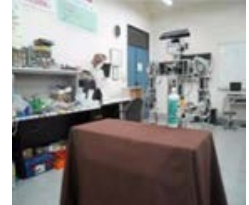

(b)

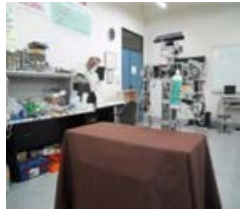

(c)
Fig. 5 Task execution of Condition A on real robot

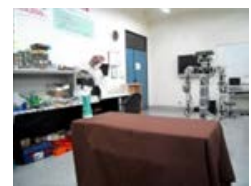

(a)

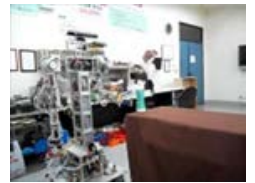

(b)

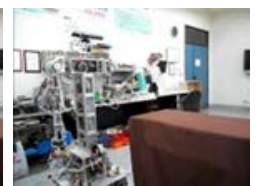

(c)
Fig. 6 Task execution of Condition B on real robot

\section{Conclusions and Future Work}

A dual-arm robotic system has been designed and implemented for mobile manipulation tasks in an unstructured environment. A grasp planning system is developed based on visual SLAM and visual servoing of the robot. The proposed system has been verified in three different conditions. The experimental results show that the robot can adapt to the variation of environment and find a suitable place to grasp the object. In the future, object fetching in more extensive environments will be studied.

\section{Acknowledgements}

This work was partly supported by Ministry of Science and Technology of Taiwan, under grant NSC 102-2221E-009-140.

\section{References}

1. Jorg Stuckler and Sven Behnke, "Benchmarking Mobile Manipulation in Everyday Environments," in Proc. of 2012 IEEE Workshop on Advanced Robotics and its Social Impacts (ARSO), (Munich, Germany, 2012), pp. 16.

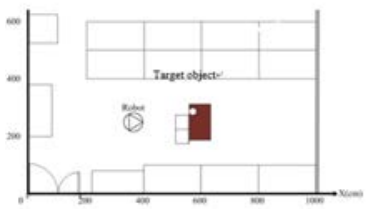

(a)

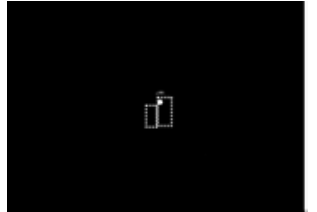

(b)
Fig. 7 (a) Environment setup of Condition C (b) the planning result of Condition C.

2. J. Klaess, J. Stuckler and S. Behnke, "Efficient Mobile Robot Navigation Using 3D Surfel Grid Maps,” in Proc. of 7th German Conf. on Robotics (ROBOTIK), (Munich, Germany, 2012), pp. 1-4.

3. Sachin Chitta, E. Gil Jones, Matei Ciocarlie and Kaijen Hsiao, "Mobile Manipulation in Unstructured Environments: Perception, Planning, and Execution,” in IEEE Robotics \& Automation Magazine, 19(2012), 5871.

4. Hornung, A., Phillips, M., Jones, E.G., Bennewitz, M., Likhachev, M. and Chitta, S., "Navigation in ThreeDimensional Cluttered Environments for Mobile Manipulation," in Proc. of 2012 IEEE International Conference on Robotics and Automation, (Minnesota, USA, 2012), pp. 423-429.

5. Freek Stulp, Andreas Fedrizzi and Michael Beetz, "Action-Related Place-Based Mobile Manipulation," in Proc. International Conference on Intelligent Robots and Systems, (St. Louis, MO, USA, 2009), pp. 3115-3120.

6. Muhammad Attamimi, Keisuke Ito, Tomoaki Nakamura and Takayuki Nagai, "A Planning Method for Efficient Mobile Manipulation Considering Ambiguity," in Proc. International Conference on Intelligent Robots and Systems, (Vilamoura-Algarve, Portugal, 2012), pp. 965972.

7. Kai-Tai Song, Sin-Yi Jiang and Chia-Chang Wu, "Pose Control of a Four-Wheel Drive Omni-Directional Mobile Robot,” in Proc. of 2012 CACS International Automatic Control Conference, ( Yunlin, Taiwan, 2012).

8. Chien-Hung Liu and Kai-Tai Song, "A New Approach to Map Joining for Depth-Augmented Visual SLAM,” in Proc. of ASCC 2013, (Istanbul, Turkey, 2013).

9. Kai-Tai Song and Shih-Cheng Tsai, "Vision-Based Adaptive Grasping of a Humanoid Robot Arm," in Proc. of IEEE ICAL 2012, (Zhengzhou, China, 2012), pp.160165. 